\title{
A Physiological Role for a Moonlighting Protein in Lipopolysaccharide- induced Endotoxemia
}

\author{
Kirsten Zborek ${ }^{1}$, Daniel Lee ${ }^{2,3,4}$, Adam Pajakowski², Jacob Slack ${ }^{4}$, Joseph Park ${ }^{4}$, \\ June Park ${ }^{4}$, Margaret Schwarz ${ }^{2,3,4}$ \\ Indiana University School of Medicine ${ }^{1}$, Indiana University School of Medicine ${ }^{2}$, \\ Department of Pediatrics, Department of Cellular and Integrative Physiology ${ }^{3}$ \\ University of Notre Dame, Department of Chemistry \& Biochemistry ${ }^{4}$
}

Background and Hypothesis: The pathogenesis of Bronchopulmonary Dysplasia (BPD) is multifactorial leading to inflammation. In BPD, EndothelialMonocyte Activating Polypeptide II (EMAP II, encoded by Aimp1), a moonlighting pro-inflammatory cytokine, is initially found in bronchiolar club cells followed by intra-alveolar GAL-3+ macrophages. Sustained EMAP II mimics BPD, invoking inflammation, alveolar simplification, and macrophage recruitment. Targeted ablation of EMAP II in the recruited macrophages may dampen innate immune response.

Experimental Design: Gender-matched, aged-matched littermate mice with myeloid-cell specific ablation of Aimp1 (Lyz2-Cre;Aimp $1^{\text {floxfflox }}$, denoted as Aimp $1^{\Delta / \Delta}$ ) or without (control) were subjected to lipopolysaccharide (LPS)endotoxemia. Survival rates of co-housed or singly housed mice were measured over 72 hours following a lethal dose $(15 \mathrm{mg} / \mathrm{kg})$. Clinical scores (0-6) based on the integrity of their locomotion, fur, and eyes were assigned every 2 hours. Blood and bone marrow smears, average bodyweights, spleen-weights to bodyweights and liver-weights to bodyweights were analyzed.

Results: There were no baseline differences in bodyweight, spleen weight:bodyweight, liver weight:bodyweight ( $p$-val $=0.42,0.46,0.64$ ). Representative bone marrow and blood smears showed no notable difference. Aimp $1^{\Delta / \Delta}$ male mice co-housed (dose $15 \mathrm{mg} / \mathrm{kg}$ ) but not singly housed survived longer than their littermates (median survival: hours); Aimp $1^{\Delta / \Delta}$ female mice showed a survival advantage (median survival: hours) with lower clinical scores than their littermates. The kinetics of NFKBIA//kB degradation was similar between $\operatorname{Aimp}^{1^{\Delta / \Delta}}$ and control peritoneal macrophages in response to LPS, although there was a higher basal amount in Aimp $1^{\Delta / \Delta}$.

Conclusion and Potential Impact: Aimp1/EMAP II does play a positive feedback role in innate immunity, potentially in a metabolically and genderspecific role of Aimp1 which remain to be explored. 